\title{
Femoral osteotomies for the valgus knee
}

\author{
Matteo Olivero ${ }^{1}$, Federica Rosso ${ }^{2}$, Federico Dettoni $^{2}$, Matteo Bruzzone ${ }^{2}$, Roberto Rossi ${ }^{1,2}$ \\ ${ }^{1}$ University of Study of Turin, Via Po 8, 10100, Turin, Italy; ${ }^{2}$ Department of Orthopedics and Traumatology, AO Ordine Mauriziano, Largo Turati \\ 62, 10128, Turin, Italy \\ Contributions: (I) Conception and design: F Rosso, F Dettoni, M Bruzzone, R Rossi; (II) Administrative support: None; (III) Provision of \\ study materials or patients: F Dettoni, M Bruzzone, R Rossi; (IV) Collection and assembly of data: M Olivero, F Rosso; (V) Data analysis and \\ interpretation: M Olivero, F Rosso, F Dettoni, M Bruzzone; (VI) Manuscript writing: All authors; (VII) Final approval of manuscript: All authors. \\ Correspondence to: Federica Rosso. AO ordine Mauriziano, Largo Turati 62, 10128, Turin, Italy. Email: federica.rosso@yahoo.it.
}

\begin{abstract}
Coronal limb malalignment is an important contributing factor to articular cartilage damage and to the development of knee osteoarthritis. Osteotomies around the knee are effective and well-recognized treatments for unloading the affected compartment. Valgus malalignment is less common than varus malalignment. Consequently, distal Femoral Osteotomies to correct the valgus knee are less commonly performed compared to high tibial osteotomy (HTO) for the varus knee. There are few papers describing the outcomes of distal femoral osteotomy (DFO) to treat valgus knee alignment and knee arthritis. In literature, there is still an open debate about the correct indication and contraindication, the best surgical technique (opening versus closing wedge), the amount of correction to achieve and the use of bone grafts to enhance osteotomies healing. However, with the correct indications, Distal Femoral Osteotomies showed good outcomes in the treatment of valgus knee. The aim of this manuscript is to review the literature about DFO in the valgus knee, summarizing the indications and contraindications, the available surgical techniques, outcomes, complications and failure rates.
\end{abstract}

Keywords: Osteotomy; valgus knee; femur; osteoarthritis

Received: 13 February 2017; Accepted: 26 May 2017; Published: 12 June 2017.

doi: 10.21037/aoj.2017.06.01

View this article at: http://dx.doi.org/10.21037/aoj.2017.06.01

\section{Introduction}

Lower limb alignment is an important contributing factor to the development of knee arthritis, and it is related to the articular cartilage wear pattern (1).

Osteotomies around the knee are a well-recognized treatment for symptomatic unicompartmental knee arthritis associated to lower limb malalignment in young and active patients, with the attempt to preserve the native knee joint and delay knee arthroplasty $(2,3)$.

Genu varum is the most common knee malalignment, and good results have been described in literature for high tibial osteotomy (HTO) performed to treat medial unicompartmental knee arthritis in varus malalignment (4).

Valgus malalignment is less common compared to varus malalignment. Excess of physiological valgus $\left(5^{\circ}-8^{\circ}\right)$ leads to mechanical overload of the lateral compartment, increasing the risk of lateral knee osteoarthritis development. Valgus deformity can be idiopathic or secondary to other pathologies (i.e., metabolic disorder, rheumatoid arthritis, rickets, poliomyelitis) or to a previous lateral tibial plateau fracture (5).

Some authors performed proximal tibial osteotomy to correct the valgus deformity but unsatisfactory results have been reported (6). This is probably because valgus deformity is often associated with hypoplastic lateral femoral condyle and with a joint line that slopes from superolaterally to inferomedially in the anterior-posterior plane. This deformity cannot be corrected unless the osteotomy is performed proximally to the joint $(6,7)$. Coventry, in 1973 , established that if valgus deformity is greater than $12^{\circ}$ or if the joint plane deviate more than $140^{\circ}$ from the horizontal one, a distal femoral osteotomy (DFO) should be performed rather than an HTO to correct the deformity (3). 
The available literature regarding DFO is limited and heterogeneous, but good clinical outcomes have been reported in different studies (8-11). However, there are still some debated issues, such as the best surgical technique (closing or opening-wedge osteotomy), the type of fixation, the amount of correction to achieve, and the need for a biological graft to fill the osteotomy gap (7).

The purpose of this article is to review the literature about DFO, trying to clarify the correct indications, outcomes and survival rates of different surgical techniques.

\section{Indications and contraindications}

The correct patient's selection is the first step to achieve good clinical outcomes after a DFO. A complete knee examination should be performed to identify pathological conditions associated with valgus deformity, such as meniscal pathology, patellofemoral diseases and ligamentous instability.

In literature there is not a unique cut-off for patient's age: for O'Malley DFO should be performed in patients younger than 55 years (12), for Tírico in patients younger than 60 years (13) and for Puddu in patients younger than 65 years (14). However, not only the age should be considered but also lifestyle, general health and the desire to stay active. Therefore, the ideal candidate for a DFO is an active patients younger than 65 years old (3).

DFO should be performed only in presence of valgus malalignment associated with isolated lateral compartment arthritis. It is still debated in literature whether patellar osteoarthritis should be considered a contraindication to perform a DFO. A varus opening wedge DFO may reduce the $\mathrm{Q}$ angle unloading the patellofemoral lateral compartment (3). Zarrouk et al., in their analysis of 26 DFOs, demonstrated that patients with patellofemoral osteoarthritis had comparable results to the others patients at the final follow-up (15). Although some authors consider symptomatic patellofemoral arthritis an absolute (13) or relative contraindication to DFO $(12,16)$, considering the theoretical mechanical advantages and Zarrouk's results, moderate patellofemoral arthritis may not be considered an absolute contraindication to $\operatorname{DFO}(15,17)$.

DFO can be associated with other concomitant procedures. Cameron et al. compared outcomes of simple DFO and DFO associated with joint preservation techniques (osteochondral allograft transplantation and/ or meniscal allograft transplantation) and demonstrated a higher survivorship in the second group (five-years survivorship: $74 \%$ in the arthritis group and $92 \%$ in the joint preservation group) (10).

In patients with valgus deformity and ligamentous instability a DFO associated with ligamentous reconstruction, in one or two stages, should be considered. As well as for HTO (18), some authors proposed DFO to treat valgus knee arthritis and instability. Hetsroni et al. experimentally demonstrated that opening wedge DFO may decrease medial opening in patients with medial collateral ligament (MCL) incompetence. They reproduced, on cadaveric specimens, progressive knee instability sequentially sectioning the superficial MCL, the deep MCL and the anterior cruciate ligament (ACL) and then evaluated valgus instability before and after opening-wedge DFO. After DFO, medial opening at $30^{\circ}$ of flexion significantly decreased when superficial MCL was transected. The authors concluded that hamstring tendons, semimembranosus tendon and the iliotibial band may play a role in the reduction of instability after DFO (19).

Pre-operative range of motion ( $\mathrm{ROM}$ ) should always be evaluated: flexion contracture greater than $15^{\circ}$ and knee flexion less than $90^{\circ}$ are usually considered contraindication to knee realignment procedures (13).

$\mathrm{DFO}$ is absolutely contraindicated in presence of tri-compartmental knee arthritis and severe articular disruption. In patients with inflammatory diseases, valgus knee deformity is common, but DFO is usually contraindicated. BMI greater than $30 \mathrm{~kg} / \mathrm{m}^{2}$ has been associated with worse outcomes after DFO (6). Other common contraindications are very unstable knee and fixed valgus deformity greater than $20^{\circ}$, because it can be associated with severe ligamentous instability. Furthermore, DFO in patients with severe osteoporosis and nicotine use could be correlated with poor outcomes (12-14,20).

Table 1 summarizes indications and contraindications to DFO.

\section{Preoperative setting}

A complete radiographic examination is mandatory to correctly plan the osteotomy. Standard radiographic assessment should include weight-bearing antero-posterior and lateral views, skyline view, Rosenberg view and a monopodalic weight-bearing long-leg view (12). The Rosenberg view is helpful to evaluate the lateral and medial compartment cartilage wear and to evaluate deformity associated with cruciate deficiency, because the cartilage wear is typically located in the posterior tibial plateau (3). 
Table 1 Indications and contraindications to distal femoral osteotomy

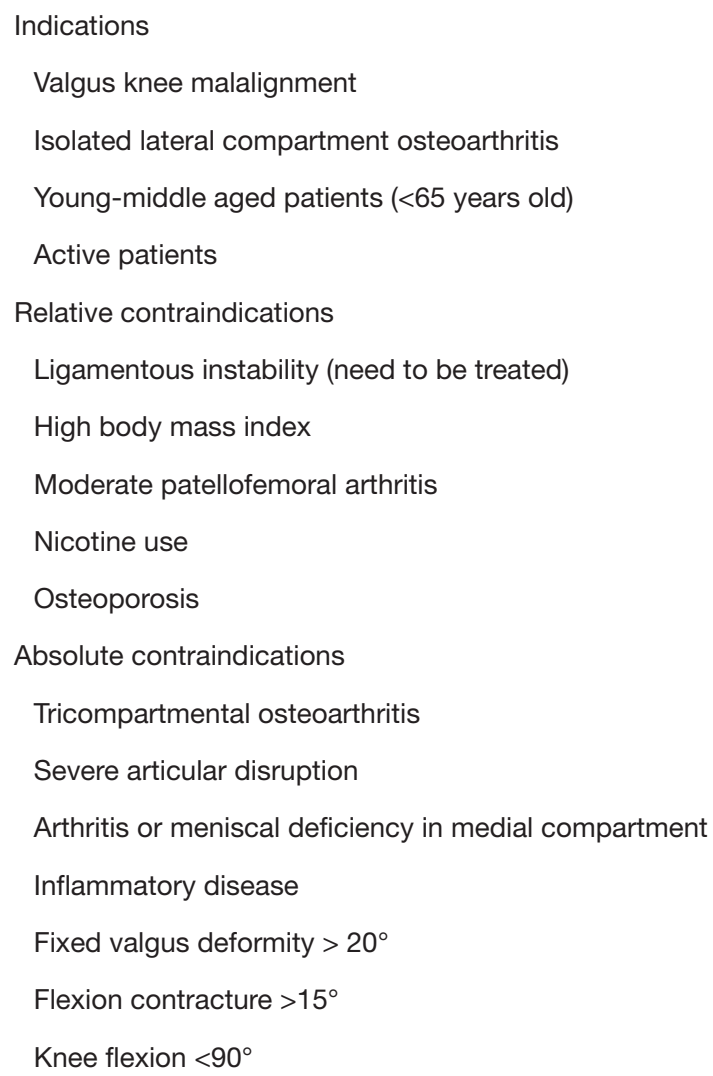

To quantify the amount of valgus deformity in the preoperative planning is possible to calculate the mechanical axis as a percentage across the tibial plateau, considering the medial edge as $0 \%$ and the lateral edge $100 \%$. Valgus is defined as mechanical axis that cross the tibial plateau beyond the $56 \%$ (20).

The amount of the correction is the angle between the mechanical axis of the femur (a line from the center of the femoral head to the center of tibial plateau) and the mechanical axis of the tibia (a line from the center of tibial plateau and the center of talar dome) (20,21). Figure 1 shows a pre-operative planning for a DFO.

In literature, there is no uniform trend about the best amount of correction to achieve. Some authors considered contraindicated overcorrection in DFO $(14,22)$. Dugdale et al. defined that the goal of DFO is mechanical axis at $48-50 \%$ of the tibial plateau (21). However, Quirno et al., in a recent biomechanical analysis, reported that over-correcting valgus deformity better unload the lateral compartment and better restore the normal biomechanics compared to correction to neutral alignment. The authors concluded

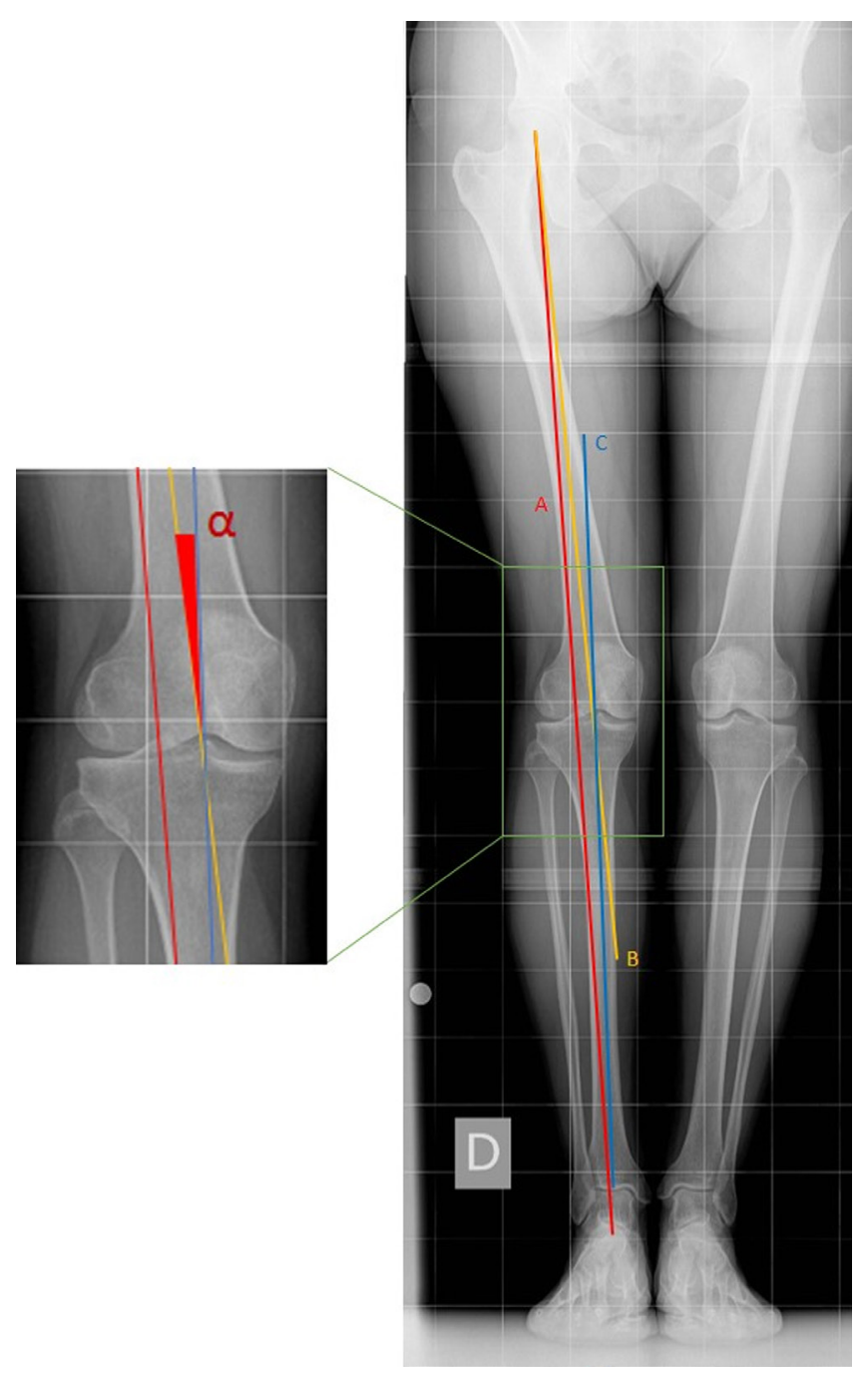

Figure 1 Preoperative planning (long leg view and focus of the knee joint on the left). (A) Mechanical axis; (B) femoral mechanical axis; (C) tibial mechanical axis. $\alpha$ : correction angle.

that overcorrecting the osteotomy of $5^{\circ}$ normalize contact pressure and contact areas in the lateral compartment (23). Similarly, others authors reported good clinical outcomes with valgus deformity over-correction (mechanical axis goal at $40-41 \%$ of tibial plateau) due to lateral compartment unloading and medial muscles' forces neutralization $(20,24)$.

The Magnetic Resonance Imaging is helpful in diagnosing any chondral or ligamentous alterations. These evaluations are mandatory to confirm the absence of osteoarthritis in the medial compartment and to plan surgical procedures associated to DFO (i.e., ligamentous reconstructions, osteochondral transplantations, meniscal suture or regularization ) $(10,14)$. 


\section{Surgical techniques}

In this section the main surgical techniques reported in literature will be briefly described: (I) medial closingwedge DFO; (II) lateral opening-wedge DFO; (III) others techniques (dome osteotomy and bi-planar osteotomy).

There is no clear indication for one technique over the others. However, O'Malley et al. contemplate the amount of correction to achieve to choose between opening- and closing-wedge osteotomy. If the correction is up to $10^{\circ}-12^{\circ}$ they prefer lateral opening-wedge osteotomy. On contrary, for correction greater than $12^{\circ}$ they usually perform medial closing-wedge osteotomy (12). However others authors consider surgeon preference and confidence with the surgical technique to indicate for a type of osteotomy over another $(13,20)$. Independently from the surgical technique, it is advisable to perform knee arthroscopy before the osteotomy. The arthroscopy allows to confirm the integrity of medial and patellofemoral compartments and to perform any intra-articular procedures such as treatment of meniscal lesions, microfractures or mosaicplasty for chondral defects $(14,25)$.

\section{Medial closing-wedge osteotomy}

A medial-side distal femoral approach is normally used with a skin incision starting $2 \mathrm{~cm}$ distal to the medial epicondyle and extending $15 \mathrm{~cm}$ proximally. The fascia over the vastus medialis is incised and retracted laterally and anteriorly to expose the femoral shaft. The osteotomy is usually performed with a saw blade in the anterior portion and with an osteotome in posterior portion, ending $10 \mathrm{~mm}$ from the lateral cortex. A blunt retractor is positioned posterior to protect neurovascular structures during the osteotomy. With the same technique a second osteotomy is performed 1 to $5 \mathrm{~mm}$ proximally to achieve the desired correction (13).

In 1988, McDermott et al. described their technique using a $90^{\circ}$ blade plate. Theoretically, it is possible to achieve $0^{\circ}$ of tibiofemoral angle positioning the $90^{\circ}$ blade parallel to the trans-epicondylar axis and the plate parallel to the medial femoral cortex (26). Healy et al. described their technique using three pins to guide the osteotomy: the first pin was placed perpendicular to the femoral shaft, $1-2 \mathrm{~cm}$ proximal to medial epicondyle. The second pin was placed at the level of the distal cut and formed, with the first one, an angle equivalent to the desired correction angle. The last pin was placed parallel to the second one but distal and anterior, and it was used as a guide for the osteotomy (27). A similar technique was described by Learmonth using dedicate jig on the anterior tibia for check the alignment (28).

Recently Forkel et al. (24) described their surgical technique: bone cuts were performed at the same way described by Healy et al. (27) but the osteotomies were fixed with locking plates. This fixation may increase the stability of the osteotomy and reduce the time of post-operative weight bearing limitation $(13,24)$.

\section{Lateral opening-wedge osteotomy}

Lateral opening-wedge DFOs have been developed more recently and they are characterized by several theoretical advantages: single bone cut, better control of correction, more anatomic correction and decreased risk of vascular injuries. Furthermore, some authors suggested that a lateral plate may acts as a tension band, with mechanical advantages compared to medial plates (14). However, there is an increased risk of malunion and non-union and potential hardware intolerance for irritation of the lateral structure of the knee (10).

Usually a $12-15 \mathrm{~cm}$ skin incision is performed from the lateral epicondyle and proximally extended. Then, the iliotibial band is incised, the vastus lateralis is elevated from inter-muscular septum and the distal femoral diaphysis and meta-diaphyseal flare are exposed (20).

If concomitant intra-articular procedures have to be performed, the approach may be extended as lateral peripatellar one (12).

Puddu et al. described their original technique developing a T-shaped tooth plate for the fixation of the osteotomy. More recently, a new version was developed allowing for locking screw fixation. With the knee in extension a guide wire is drilled from 2-3 fingerbreadths above to the lateral epicondyle to few millimeters proximal the medial epicondyle, with an inclination of about $20^{\circ}$ (14). The osteotomy is performed in the lateral portion with an oscillating saw blade and then carefully completed with osteotomes preserving at least $1 \mathrm{~cm}$ of medial cortex. Then, the osteotomy can be gradually opened until the desired correction is achieved $(12,14)$. The osteotomy is stabilized with the plate with the "tooth" of the same size of the wedge, and it is fixed with screws. Post-operatively, the authors suggested immobilizing the knee in full extension or $10^{\circ}$ of flexion when standing. The patients are kept nonweight-bearing for 6 weeks, then partial weight-bearing is allowed for 2 weeks and full weight-bearing is allowed 8 weeks after surgery, after radiological confirmation of 

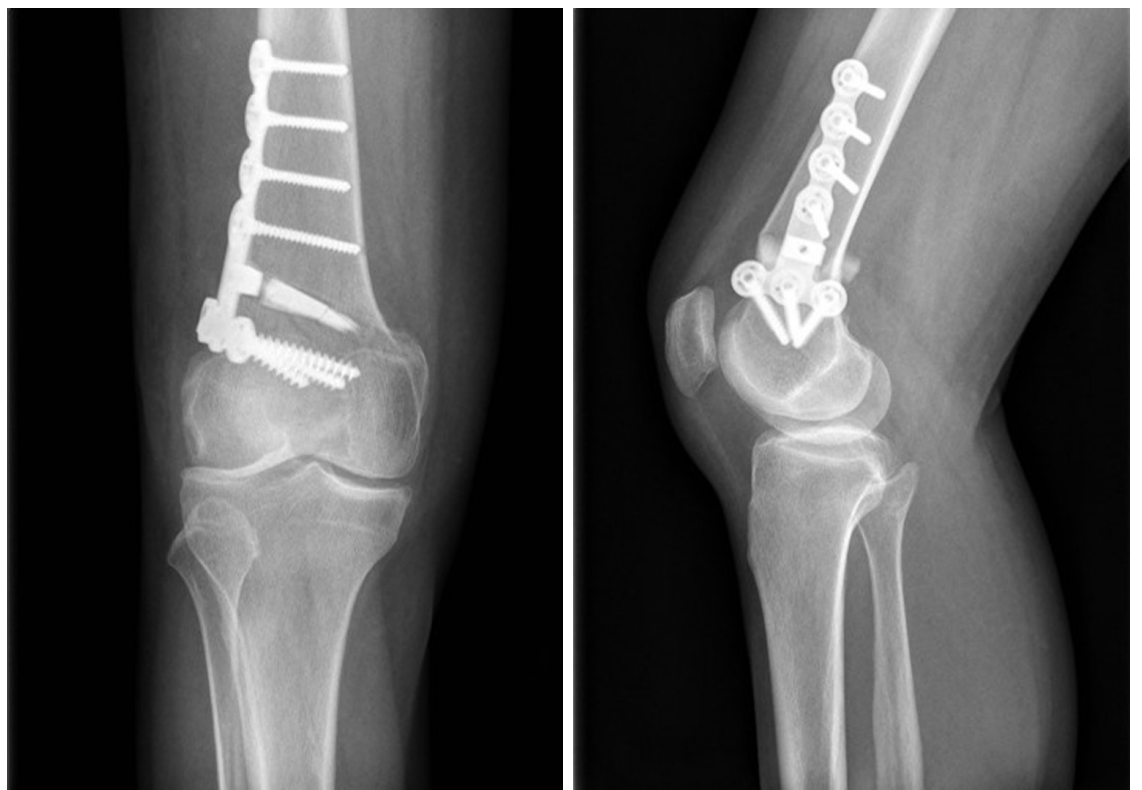

Figure 2 Post-operative X-rays (AP view on the left and lateral view on the right) of a lateral opening-wedge DFO stabilized using a Puddu Plate.

osteotomy healing (14). Figure 2 shows a post-operative $\mathrm{x}$-ray of a lateral opening wedge osteotomy.

Recently, other authors described similar surgical techniques using the TomoFix Plate (Synthes $\left.{ }^{\circledR}\right)(12,20,29)$. This fixation system is more stable compared to Puddu plate, allowing for early weight bearing. Some authors described an increased risk of soft tissue irritation using the Tomofix plate (30). However, no clinical differences were reported in literature.

In literature, the use of graft to fill the gap in openingwedge osteotomy is still debated. Puddu used tri-corticocancellous graft from the ipsilateral iliac crest in gaps greater than $7.5 \mathrm{~mm}$ (14). Many grafts have been described: bone allograft, bone autograft and synthetic bone substitution (bone cement, hydroxyapatite, $\beta$-tricalcium phosphate). These grafts can be enhanced with plateletrich-plasma (PRP), growth factors, and bone marrow stromal cells. Encouraging results have been reported with these techniques but they are still experimental (31). Iliac crest autograft is still considered the gold standard but, considering donor site morbidity, bone substitutes may be a good option, despite the concerns about mechanical properties and biological degradability.

In recent descriptions of their surgical techniques Mitchell (20) suggested the use of cancellous demineralized bone matrix allograft while O'Malley (12) reported the employ of wedges harvested from femoral head allograft.
However, the authors did not describe any comparison with other grafts.

\section{Others surgical techniques}

Dome osteotomies are cylindrical osteotomies that rotate around a central axis. Using a Center Of Rotation of Angulation (CORA) at the level of the knee joint is possible to perform a distal femoral focal dome osteotomy achieving deformity correction without leg length variations. Moreover, bone cuts are completely congruent with big contact surfaces allowing increased healing rate (32). Nevertheless, only few reports in literature described the outcomes of femoral dome osteotomies.

In 2010 Brinkman et al. described bi-planar osteotomy. The theoretical advantage is to create a larger surface at the site of osteotomy with increased stability and stiffness (25). The bone cuts are performed similarly to a medial closingwedge osteotomy, but only in the posterior three fourths of the femur. The osteotomy is then completed with an additional oblique ascending bone cut on the anterior surface of the femur (25).

Brinkman et al. in their biomechanical study confirmed an improvement in axial stability but a decreased stability under torsional loading using this technique (33). Recently good clinical outcomes with biplanar osteotomy were reported in literature (7). 
Saragaglia et al. performed a varus producing DFO with computer assisted navigation to obtain a more accurate correction of the deformity. The authors reported satisfactory results with $86 \%$ of congruence with the preoperative planning goals (34).

\section{Complications}

The correct patients' selection and evaluation of surgical risk are mandatory in preventing complications after DFO (35).

Injury to the popliteal neurovascular bundle is the most severe complication of DFO but fortunately the rate of occurrence is very low, as described by Georgoulis et al. (2 vascular injuries in 250 patients) (36,37). Kim et al. analyzed the distance between the popliteal artery and the posterior cortex of the tibia in fresh-frozen cadaveric lower extremities, concluding that neurovascular structures are farther from the tibia with the knee at $90^{\circ}$ of flexion (36). Most of the authors suggest performing the osteotomy with the knee flexed, with attention to the position of blunt retractors to avoid neurovascular damages $(12,14,20)$.

Intra-operatively, surgeons must be careful also to uncontrolled propagation of fractures through the articular surface or the far cortex. If the pin is positioned too close to the joint or if the surgeon does not leave enough bone on the medial hinge, the fracture risk increases significantly. This kind of injury is more frequent in opening wedge technique compared to closing wedge. When medial hinge disruption occurs the surgeon can fix it with a contralateral screw or staple (14).

Rates of major post-operative complications are comparable with HTO and total knee prosthesis (3). Willey et al. (38) reported $20 \%$ of major complications in 78 patients (joint contracture 9\%, hardware failure 9\% compartment syndrome and loss of correction $2 \%$, intra-articular fractures $4 \%$ ). Deep venous thrombosis is a relatively common complication with greater risk 3-4 days after surgery and is reported ranging from $0 \%$ to $10.8 \%$ (35).

With respect to others postoperative complications, heterogeneous results have been reported in literature. Edgerton et al. analyzed 24 patients and observed non-union in $25 \%$ of patients and loss of correction in 21\% (39). Mathews et al. reported that $57 \%$ of 21 patients had complication after DFO: severe knee stiffness (48\%), non-union-delayed union (19\%), infection (10\%) and fixation failure (5\%) (40). Jacobi et al. analyzed 14 patients treated with opening wedge DFO fixed with TomoFix Plate and they described high rate of delayed osteotomy healing. All of the osteotomies were not healed at 6 weeks, only $50 \%$ had sufficient consolidation at 3-month and, at 6-month, $14 \%$ of patients showed persisting insufficient healing. They did not find any significant correlations between the amount of correction or the use of bone graft and the delayed consolidation of the osteotomy. They also reported plate intolerance in $86 \%$ of patients. For these reasons this procedure has been abandoned by the authors and they adopted closing wedge technique as treatment of choice (29).

If we analyze more recent papers in literature nonunion trend is decreasing. Ekeland et al. reported that in their study $75 \%$ of 24 osteotomies healed in 3 months and the rest within 6 months (30). Forkel et al. observed that $5 \%$ of the patients had revision surgery for delayed union or nonunion of the osteotomy (24). Most of the authors reported higher non-union rate in opening-wedge than in closing wedge DFOs $(22,24,30)$.

Hardware intolerance in opening-wedge DFO is a frequent complication: Saithna et al reported that $76 \%$ of 21 patients underwent further surgery and the most common of which was plate removal (22). Ekeland et al. reported that less than half of Puddu Plate in their study had to be removed for tissue irritation (30). The TomoFix Plate is larger and more stable than the Puddu Plate but it gives more local soft tissue irritation and has frequently to be removed. The high frequency of hardware removal should be preoperatively discussed with patients (10).

Post-operative infection is another possible complication. Anagnostakos et al. reported, in their review, a comprehensive rate of superficial infection from $1 \%$ to $9 \%$ and deep infection from $0.5 \%$ to $4.7 \%$ (41). Infections are more common with external fixation compared to other techniques, pin tract infections are reported between $2 \%$ and $71 \%$, most of them can be managed with antibiotic therapy (35).

\section{Results}

In literature there are limited and highly heterogeneous papers describing the outcomes of both opening and closing-wedge DFO (7).

Good results are reported for closing wedge DFOs at 10 years of follow up with survival rates ranging between $64 \%$ (42) and $89.9 \%$ (43). Finkelstein et al. (42) evaluated 24 DFOs and reported one of the lowest survival rate $(64 \%$ at 133 months) but this analysis was performed in 1996 with older surgical technique and fixation methods. Few studies described longer follow-up: Backstein et al. (40 closing 
wedge DFOs) (8) reported 15-year survival rate of $45 \%$ while Kosashvili et al. (36 closing wedge DFOs) (9) reported survival rate of $52.5 \%$ at the same follow-up. A recent review confirm the significantly increasing of failure rates in follow-up longer than 10 years (7).

Good survival rate at 5-year follow-up are reported in literature also for opening-wedge DFO, ranging from $74 \%$ to $100 \%(11,15,22,34,44,45)$. Cameron et al. compared outcomes of DFO (19 knees) and DFO associated with joint preservation techniques (12 knees), and reported a survival rate of $74 \%$ at 5 -year follow up in the first group compared to $92 \%$ in the second group (10).

Ekeland et al. (30) evaluated 24 opening wedge DFOs and concluded with $74 \%$ of survival rate at 10 years of follow-up. Forkel et al. described 23 DFOs and reported a significant increase in all sub-items of KOOS (Knee Injury and Osteoarthritis Outcome Score) with closing-wedge technique (24).

Similar results are reported also for patients treated with opening-wedge DFOs. Zarrouk et al. described a significant improvement in three different scores in 22 patients (International Knee Society score-IKS, modified KSS and Functional Score) (15). Ekeland et al. reported that 1 year after surgery the KOOS score increased significantly for each sub-score. However, this score remained at the same level for all patients with survived osteotomy at final follow-up (30).

Honorio de Carvalho et al. evaluated 26 V-shaped DFOs: $88.5 \%$ of patients returned to normal work activity and 14 out of 15 active individuals resumed their physical activity (46).

Direct comparisons between different surgical techniques are difficult, because most of the studies utilized different scores. Apparently, there is no clear superiority of a surgical technique over the other, and the choice remains largely surgeon dependent.

The method of fixation could be a factor that considerably affects the results of the osteotomy. Mathews et al. described results of 21 closing wedge DFOs performed with different method of fixation: plaster cast, staples and internal fixation. They concluded that using an internal rigid fixation is fundamental in obtaining good results and post-operative early mobilization (40). Recently, Kazemi et al. compared 20 DFOs fixed with Angle Blade Plate and 20 DFOs fixed with Locking Compression Plate. All the osteotomies in the first group healed within nine months while there were four cases of non-union in the second group. They concluded that this difference was not statistically significant but seems that it could be significant if more patients were enrolled in the study (47). Brinkman et al. in 2011 compared five different configurations of osteotomy and fixation methods on composite femur models simulating physiological loading. The authors analyzed: oblique medial closing-wedge osteotomy fixed with angle-stable plate (TomoFix Plate, Synthes) and fixed with $90^{\circ}$ angled-blade plate (AO), perpendicular medial closing-wedge osteotomy fixed with $90^{\circ}$ angled-blade plate (AO), lateral opening-wedge osteotomy fixed with spacer plate (Arthrex Spacer Plate) and fixed with angled-stable plate (TomoFix Plate, Synthes). They reported that angledblade plates are characterized by superior stability and stiffness compared to angle-stable plate and the association between oblique osteotomy and angled-blade plate was the most stable configuration. The authors concluded that there is not a clear recommendation for using an angle-stable plate but there are biological and surgical advantages such as secondary bone healing and less demanding technique (33). Tables 2,3 summarized all the studies about opening and closing wedge osteotomies.

In patients with failed DFOs, performing a total knee arthroplasty (TKA) is an highly demanding procedure with an increased risk of poor results (7). In literature only few authors reported the outcomes of TKA after DFO (48). DFO results in the creation of an extra-articular deformity close to the knee with relative proximal translation of the medial femoral condyle. Because of this, it is often required a greater amount of resection from the lateral condyle to achieve proper balance for prostheses implantation (49). Sometimes, intra-articular correction may lead to ligamentous instability not correctable with ligament release and constrained condylar prostheses are needed (48). Moreover, the displacement of the intercondylar notch, may lead to a challenging identification of the entry point of intramedullary guide $(48,49)$.

On the basis of higher infection rate $(10 \%)$ reported in TKA performed in post-traumatic knee arthritis (50), Nelson et al. recommended preoperative joint aspiration, intraoperative gram-staining, frozen section analysis and the use of antibiotic-impregnated cement in all patients undergoing TKA following DFO (48).

Despite of an high demanding surgical technique, Nelson et al. and Kosashvili et al., in their analysis of respectively 11 and 22 TKA after DFO, reported a statistically significant increase in Knee Society Score and Knee Function Score but functional benefits of arthroplasty may be limited as compared with primary arthroplasty $(35,48,49)$. 


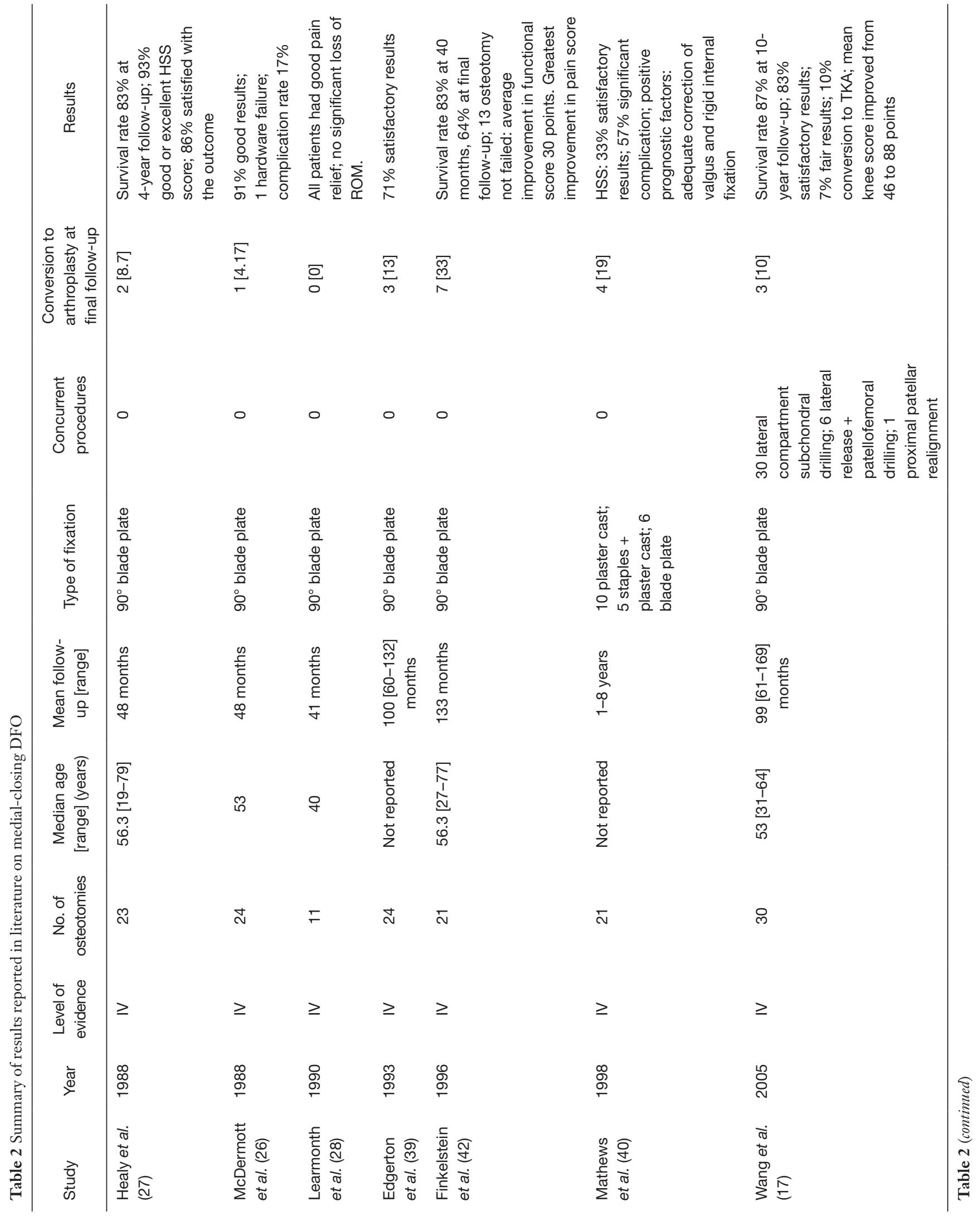




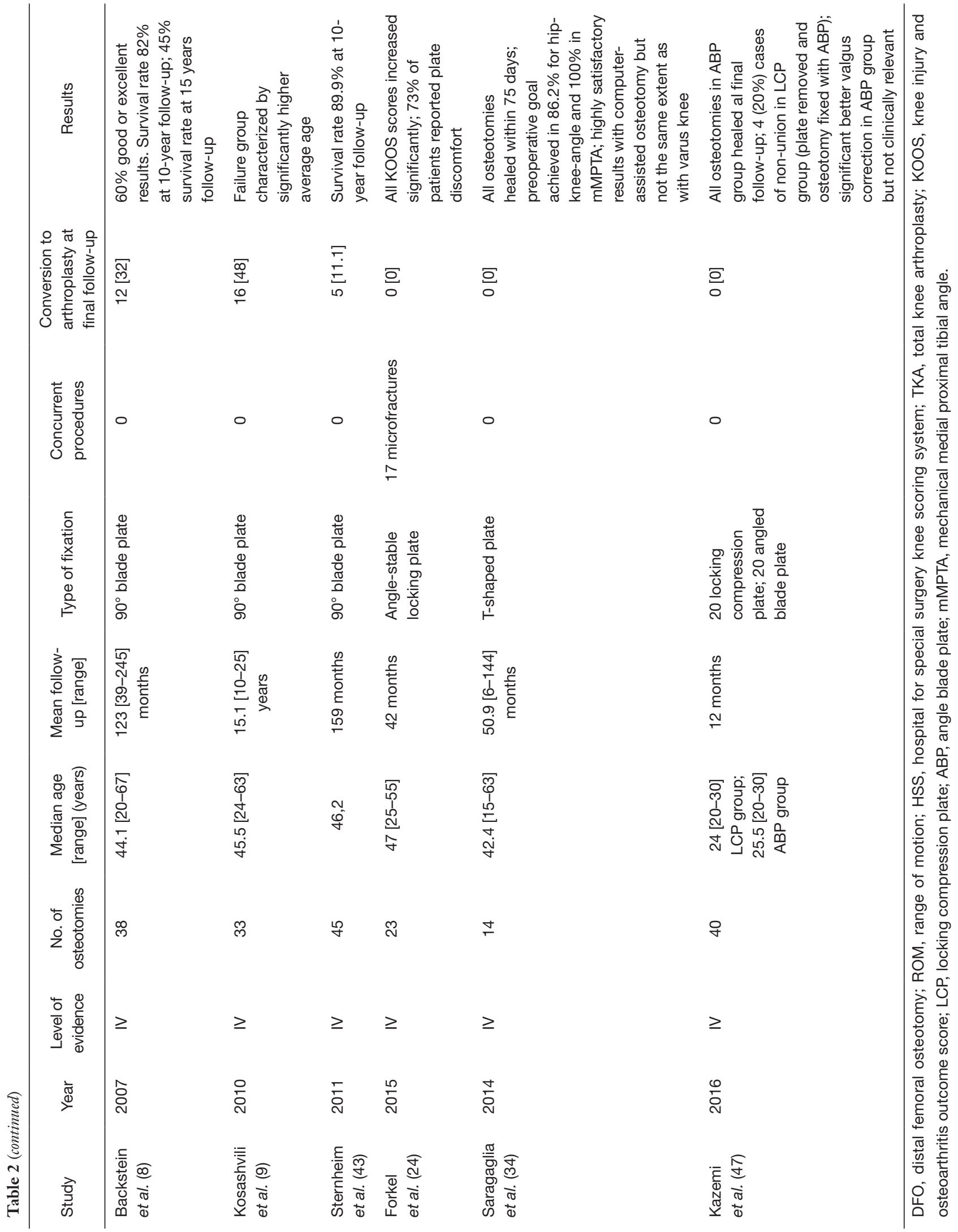




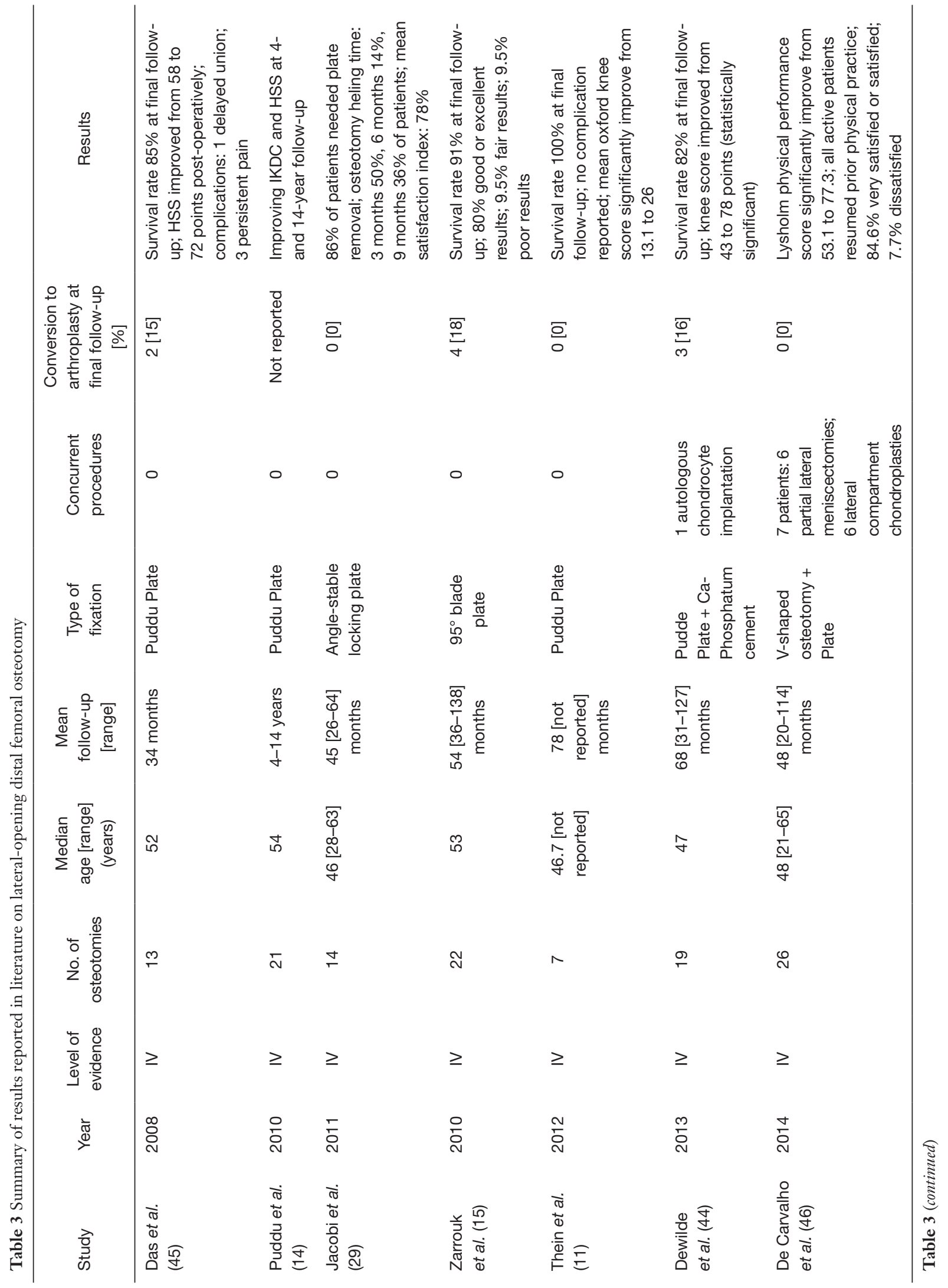




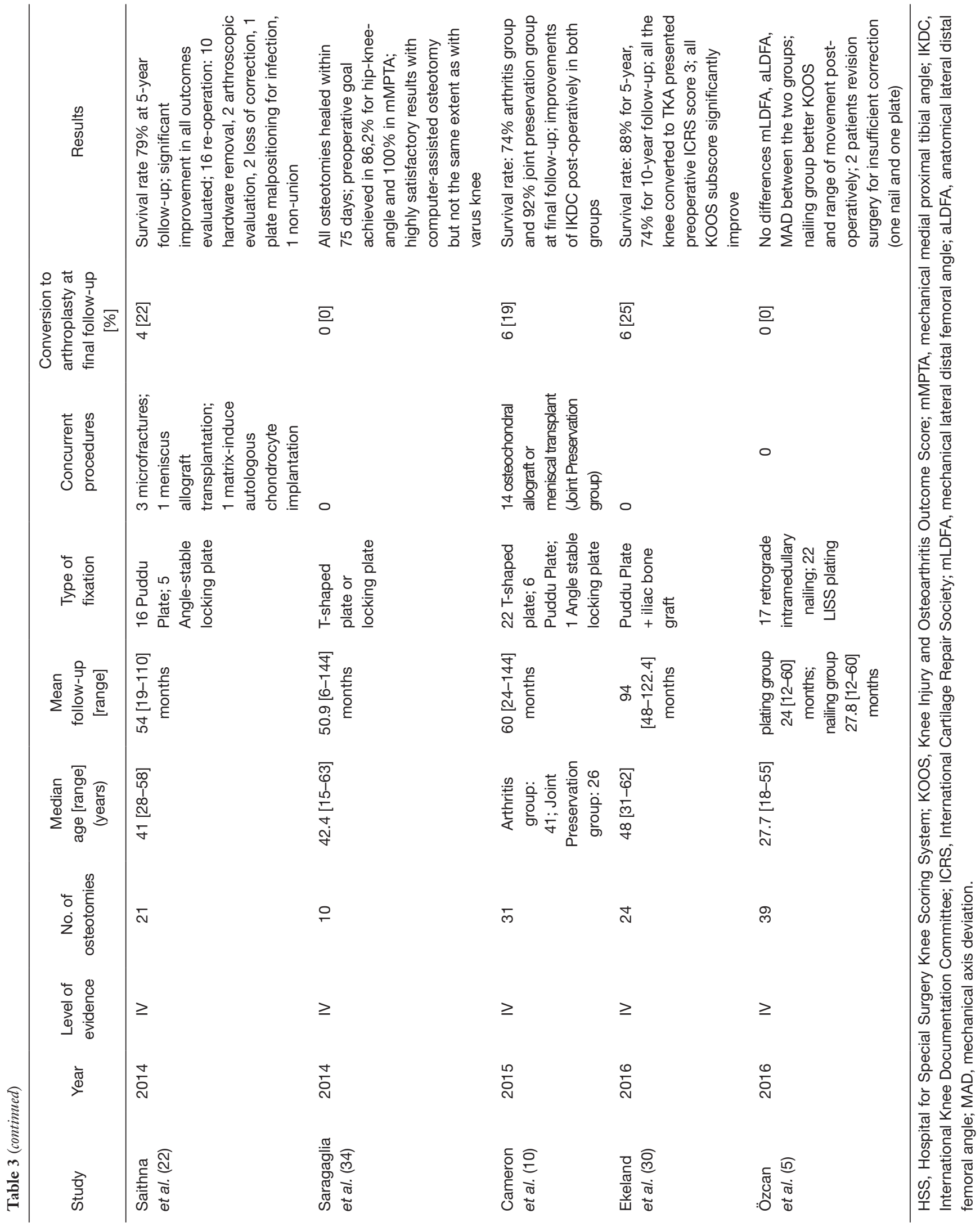




\section{Conclusions}

Fewer reports describing DFO are available in literature compared with HTO, probably because valgus deformity is less common. The main indication to DFO is valgus deformity of the knee and isolated lateral knee arthritis in young and active patients. DFOs are normally contraindicated in presence of inflammatory diseases, tricompartmental arthritis, severe articular disruption, valgus deformity greater than $20^{\circ}$, flexion contracture $>15^{\circ}$ and knee flexion $<90^{\circ}$. DFO has most often been performed with medial closing-wedge, with survival rates ranging between $95 \%(26)$ at 4 -year and $52.5 \%$ (9) at 15 -year follow-up.

Because of success of opening wedge HTO, different authors began to perform lateral opening-wedge DFOs, with survival rate ranging from $100 \%$ (11) at 6-year to $74 \%$ (30) at 10-year follow-up.

Opening-wedge technique has the advantage of being easier to perform and allowing for a more precise correction, but there is an increased risk of non-union compared with closing-wedge technique. Nowadays, in literature, there is no clear superiority of one surgical technique over the others, and the osteotomy choice is largely surgeon dependent $(2,7)$.

Recently, the interest in osteotomy increased for the possibility of association with cartilage restoration surgery (osteochondral or meniscus allograft), with possible better results compared to DFO alone (10). One of the issues about DFOs is the method of osteotomy fixation, which may influence the outcomes. Different authors stated that a stable fixation is mandatory to achieve osteotomy healing (40), metal plates are surely effective in obtaining rigid and stable fixation, but they require for extensive soft tissue damage. Conversely, external fixation is less invasive and allows for a gradual correction, but in site infection, restriction of ROM and discomfort are common $(35,41)$. There are still some open debates in literature about the amount of correction to achieve, the use of bone grafts and post-operative protocol. Some authors consider contraindicated varus overcorrection $(14,22)$ while others authors reported good clinical outcomes overcorrecting the mechanical axis (24).

Different bone graft used to fill the gap after openingwedge DFO are described in literature: allograft, autograft and synthetic bone substitution. Despite the absence of comparative studies, some authors suggest to use a bone graft to fill gap greater than $7.5 \mathrm{~mm}$ (14). There are no studies comparing the healing rate using autograft, allograft or bone substitute.

With respect to post-operative programs, patients are usually kept non weight-bearing or partial weight-bearing until the osteotomy healing. Some authors allow early weight bearing using biplanar osteotomy (33) or fixing opening-wedge osteotomy with TomoFix Plate (12).

In conclusion, DFO seems to be a reasonable option to treat painful genu valgum with association of lateral compartment arthritis in young or middle-aged patients. There are no proofs about the superiority of a surgical technique over the others. Both techniques showed good outcomes: the choice of one or another in dependent by the confidence of the surgeon.

TKA after a failed DFO presents more surgical challenges than a standard case, nevertheless showed satisfactory but inferior results compared to standard primary knee replacement, with an increased risk of complications and infection.

\section{Acknowledgements}

None.

\section{Footnote}

Conflicts of Interest: Roberto Rossi is a teaching consultant for Zimmer Biomet ${ }^{\circledR}$. The others authors have no conflicts of interest to declare.

\section{References}

1. Sharma L, Song J, Felson DT, et al. The Role of Knee Alignment in Disease Progression and Functional Decline in Knee Osteoarthritis. JAMA 2001;286:188-95.

2. Wylie JD, Jones DL, Hartley MK, et al. Distal Femoral Osteotomy for the Valgus Knee: Medial Closing Wedge Versus Lateral Opening Wedge: A Systematic Review. Arthroscopy 2016;32:2141-7.

3. Rosso F, Margheritini F. Distal femoral osteotomy. Curr Rev Musculoskelet Med 2014;7:302-11.

4. Bonasia DE, Dettoni F, Sito G, et al. Medial opening wedge high tibial osteotomy for medial compartment overload/arthritis in the varus knee: prognostic factors. Am J Sports Med 2014;42:690-8.

5. Özcan Ç, Sökücü S, Beng K, et al. Prospective comparative study of two methods for fixation after distal femur corrective osteotomy for valgus deformity; retrograde 
intramedullary nailing versus less invasive stabilization system plating. Int Orthop 2016;40:2121-6.

6. Coventry MB. Proximal tibial varus osteotomy for osteoarthritis of the lateral compartment of the knee. J Bone Joint Surg Am 1987;69:32-8.

7. Chahla J, Mitchell JJ, Liechti DJ, et al. Openingand Closing-Wedge Distal Femoral Osteotomy: A Systematic Review of Outcomes for Isolated Lateral Compartment Osteoarthritis. Orthop J Sports Med 2016;4:2325967116649901.

8. Backstein D, Morag G, Hanna S, et al. Long-term follow-up of distal femoral varus osteotomy of the knee. J Arthroplasty 2007;22:2-6.

9. Kosashvili Y, Safir O, Gross A, et al. Distal femoral varus osteotomy for lateral osteoarthritis of the knee: a minimum ten-year follow-up. Int Orthop 2010;34:249-54.

10. Cameron JI, McCauley JC, Kermanshahi AY, et al. Lateral Opening-wedge Distal Femoral Osteotomy: Pain Relief, Functional Improvement, and Survivorship at 5 Years. Clin Orthop Relat Res 2015;473:2009-15.

11. Thein R, Bronak S, Thein R, Haviv B. Distal femoral osteotomy for valgus arthritic knees. J Orthop Sci 2012;17:745-9.

12. O'Malley MP, Pareek A, Reardon PJ, et al. Distal Femoral Osteotomy: Lateral Opening Wedge Technique. Arthrosc Tech 2016;5:e725-30.

13. Tírico LE, Demange MK, Bonadio MB, et al. Medial Closing-Wedge Distal Femoral Osteotomy: Fixation With Proximal Tibial Locking Plate. Arthrosc Tech 2015;4:e687-95.

14. Puddu G, Cipolla M, Cerullo G, et al. Which osteotomy for a valgus knee? Int Orthop 2010;34:239-47.

15. Zarrouk A, Bouzidi R, Karray B, et al. Distal femoral varus osteotomy outcome: Is associated femoropatellar osteoarthritis consequential? Orthop Traumatol Surg Res 2010;96:632-6.

16. Stähelin T, Hardegger F, Ward JC. Supracondylar Osteotomy of the Femur with Use of Compression. Osteosynthesis with malleable implant. J Bone Joint Surg Am 2000;82:712-22.

17. Wang JW, Hsu CC. Distal Femoral Varus Osteotomy for Osteoarthritis of the Knee. J Bone Joint Surg Am. 2005;87:127-33.

18. Savarese E, Bisicchia S, Romeo R, et al. Role of high tibial osteotomy in chronic injuries of posterior cruciate ligament and posterolateral corner. J Orthop Traumatol 2011;12:1-17.

19. Hetsroni I, Lyman S, Pearle AD, et al. The effect of lateral opening wedge distal femoral osteotomy on medial knee opening: clinical and biomechanical factors. Knee Surg Sports Traumatol Arthrosc 2014;22:1659-65.

20. Mitchell JJ, Dean CS, Chahla J, et al. Varus-Producing Lateral Distal Femoral Opening-Wedge Osteotomy. Arthrosc Tech 2016;5:e799-807.

21. Dugdale TW, Noyes FR, Styer D. Preoperative Planning for High Tibia1 Osteotomy The Effect of Lateral Tibiofemoral Separation and Tibiofemoral Length. Clin Orthop Relat Res 1992;(274):248-64.

22. Saithna A, Kundra R, Getgood A, et al. The Knee Opening wedge distal femoral varus osteotomy for lateral compartment osteoarthritis in the valgus knee. Knee 2014;21:172-5.

23. Quirno M, Campbell KA, Singh B, et al. Distal femoral varus osteotomy for unloading valgus knee malalignment : a biomechanical analysis. Knee Surg Sports Traumatol Arthrosc 2017;25:863-8.

24. Forkel P, Achtnich A, Petersen W. Midterm results following medial closed wedge distal femoral osteotomy stabilized with a locking internal fixation device. Knee Surg Sports Traumatol Arthrosc 2015;23:2061-7.

25. Brinkman JM, Hurschler C, Staubli AE, et al. Axial and torsional stability of an improved single-plane and a new bi-plane osteotomy technique for supracondylar femur osteotomies. Knee Surg Sports Traumatol Arthrosc 2011;19:1090-8.

26. McDermott AG, Finklestein JA, Farine I, et al. Distal Femoral for Valgus of the Knee. J Bone Joint Surg Am 1988;70:110-6.

27. Healy WL, Anglen JO, Wasilewski SA, et al. Distal femoral varus osteotomy. J Bone Joint Surg Am 1988;70:102-9.

28. Learmonth ID. A simple technique for varus supracondylar osteotomy in genu valgum. J Bone Joint Surg Br 1990;72:235-7.

29. Jacobi M, Wahl P, Bouaicha S, et al. Distal femoral varus osteotomy: problems associated with the lateral open-wedge technique. Arch Orthop Trauma Surg 2011;131:725-8.

30. Ekeland A, Nerhus TK, Dimmen S, Heir S. Good functional results of distal femoral opening-wedge osteotomy of knees with lateral osteoarthritis. Knee Surg Sports Traumatol Arthrosc 2016;24:1702-9.

31. Amendola A, Bonasia DE. Results of high tibial osteotomy: review of the literature. Int Orthop 2010;34:155-60.

32. El Ghazaly SA, El-Moatasem el-HM. Femoral supracondylar focal dome osteotomy with plate fixation for acute correction of frontal plane knee deformity. Strategies 
Trauma Limb Reconstr 2015;10:41-7.

33. Brinkman JM, Hurschler C, Agneskirchner JD, et al. Axial and torsional stability of supracondylar femur osteotomies: biomechanical comparison of the stability of five different plate and osteotomy configurations. Knee Surg Sports Traumatol Arthrosc 2011;19:579-87.

34. Saragaglia D, Chedal-Bornu B. Computer-assisted osteotomy for valgus knees: medium-term results of 29 cases. Orthop Traumatol Surg Res 2014;100:527-30.

35. Vena G, Adamio SD, Amendola A. Complications of Osteotomies about the Knee. Sports Med Arthrosc 2013;21:113-20.

36. Kim J, Allaire R, Harner CD. Vascular safety during high tibial osteotomy: a cadaveric angiographic study. Am J Sports Med 2010;38:810-5.

37. Georgoulis AD, Makris C, Papageorgiou CD, et al. Nerve and vessel injuries during high tibial osteotomy combined with distal fibular osteotomy : a clinically relevant anatomic study. Knee Surg Sports Traumatol Arthrosc 1999;7:15-9.

38. Willey M, Wolf BR, Kocaglu B, et al. Complications Associated with Realignment Osteotomy of the Knee Performed Simultaneously with Additional Reconstructive Procedures. Iowa Orthop J 2010;30:55-60.

39. Edgerton BC, Mariani EM, Morrey BF. Distal femoral varus osteotomy for painful genu valgum. A fiveto-11-year follow-up study. Clin Orthop Relat Res $1993 ;(288): 263-9$.

40. Mathews J, Cobb AG, Richardson S, et al. Distal femoral osteotomy for lateral compartment osteoarthritis of the knee. Orthopedics 1998;21:437-40.

41 Anagnostakos K, Mosser P, Kohn D. Infections after high tibial osteotomy. Knee Surg Sports Traumatol Arthrosc 2013;21:161-9.

doi: 10.21037/aoj.2017.06.01

Cite this article as: Olivero M, Rosso F, Dettoni F, Bruzzone M, Rossi R. Femoral osteotomies for the valgus knee. Ann Joint 2017;2:31.
42. Finkelstein JA, Gross AE, Davis A. Varus Osteotomy of the Distal Part of the Femur. A survivorship analysis.J Bone Joint Surg Am 1996;78:1348-52.

43. Sternheim A, Garbedian S, Backstein D. Distal femoral varus osteotomy: unloading the lateral compartment: longterm follow-up of 45 medial closing wedge osteotomies. Orthopedics 2011;34:e488-90.

44. Dewilde TR, Dauw J, Vandenneucker H, et al. Opening wedge distal femoral varus osteotomy using the Puddu plate and calcium phosphate bone cement. Knee Surg Sports Traumatol Arthrosc 2013;21:249-54.

45. Das D, Sijbesma T, Hoekstra H, et al. Distal femoral opening-wedge osteotomy for lateral compartment osteoarthritis of the knee. Open Access Surg 2008;(1):25-9.

46. de Carvalho LH Jr, Temponi EF, Soares LF, et al. Physical activity after distal femur osteotomy for the treatment of lateral compartment knee osteoarthritis. Knee Surg Sports Traumatol Arthrosc 2014;22:1607-11.

47. Kazemi SM, Minaei R, Safdari F, et al. Supracondylar Osteotomy in Valgus Knee: Angle Blade Plate Versus Locking Compression Plate. Arch Bone Jt Surg 2016;4:29-34.

48. Nelson CL, Saleh KJ, Kassim RA, et al. Total Knee Arthroplasty After Varus Osteotomy of the Distal Part of the Femur. J Bone Joint Surg Am 2003;85-A:1062-5.

49. Kosashvili Y, Gross AE, Zywiel MG, et al. Total knee arthroplasty after failed distal femoral varus osteotomy using selectively stemmed posterior stabilized components. J Arthroplasty 2011;26:738-43.

50. Lonner JH, Pedlow FX, Siliski JM. Total knee arthroplasty for post-traumatic arthrosis. J Arthroplasty 1999;14:969-75. 